\title{
Le syndrome de Turcot à l'heure des gènes de prédisposition aux cancers
}

En 1959, Turcot et al. (Québec, Canada) décrivaient chez un frère et une sœur atteints de polypose adénomateuse du côlon, le développement de tumeurs malignes du système nerveux central, un médulloblastome pour l'un, un glioblastome cérébral et un adénome hypophysaire pour l'autre [1]. Depuis, plus de cent vingt cas ont été décrits, incluant un large spectre de tumeurs recto-coliques allant du simple adénome à la polypose adénomateuse typique et des tumeurs primitives du système nerveux central de types histologiques divers. Le mode de transmission, autosomique récessif ou dominant, reste controversé.

Aujourd'hui, ont été caractérisés sur le plan génétique deux syndromes malins familiaux colorectaux: la polypose adénomateuse colorectale familiale, due à une anomalie germinale du gène $A P C \quad\left(m / s n^{\circ} 7\right.$, vol. 7 , p. 718) [2], et le syndrome de Lynch ou HNPCC (pour hereditary non polyposis colon cancer) [3]. Le gène $A P C$ code pour une protéine considérée comme un suppresseur de tumeur. Sa fonction est encore mal connue; elle règle peut-être l'interaction entre la E-cadhérine et les caténines et jouerait un rôle dans la transmission du signal ( $m / s n^{\circ} 2$, vol. 10, p. 228) [4]. Le syndrome de Lynch, quant à lui, peut être provoqué par une mutation germinale sur l'un des gènes d'une famille impliquée dans la réparation de l'ADN et le maintien de l'intégrité du génome, hMSH2, hMLH1, hPMS1 et hPMS2 [4]. Dans les tumeurs de l'HNPCC, on observe le plus souvent des erreurs de réplication et une instabilité des microsatellites. La question se posait de savoir si l'un ou plusieurs de ces gènes pouvait être en cause dans le syndrome de Turcot.
Toronto et Québec, Canada) rapportent une étude portant sur quatorze familles ayant chacune au moins un malade avec une tumeur primitive du cerveau et, soit des adénomes colorectaux multiples, soit une histoire familiale de polypose adénomateuse colorectale [5]. Ces malades ont été sélectionnés à partir des registres des cancers colorectaux de Toronto (trois familles à partir de deux cent trente familles inscrites au registre) et de Johns Hopkins (onze familles sur cinq cent dix-sept). Le diagnostic clinique entre polypose adénomateuse et syndrome de Lynch est parfois très difficile à établir car certaines polyposes ne comportent que de rares adénomes. Douze familles présentaient les critères cliniques de polypose (polypes adénomateux coliques multiples). Deux familles comportaient des malades avec adénomes et carcinome coliques, sans signe évident de polypose.

Le gène $A P C$ a été étudié chez au moins un membre atteint de chaque famille. Parmi les douze familles classées initialement dans le groupe des polyposes familiales, des mutations du gène $A P C$ furent trouvées dix fois, qui toutes spécifiaient des protéines APC tronquées. Dans deux familles, aucune anomalie du gène $A P C$ n'a été mise en évidence, non plus que chez les deux familles classées dans le groupe présumé HNPCC. Les tumeurs cérébrales étaient surtout à type de médulloblastome chez les porteurs de mutation du gène $A P C$ $(80 \%)$, mais deux malades avaient un astrocytome et l'un un épendymome. La tumeur cérébrale avait suivi le diagnostic de polypose colique chez quatre malades et l'avait précédé chez six.

Chez trois malades (dont l'un classé initialement dans le goupe des poly- poses adénomateuses familiales), plusieurs tumeurs étaient retrouvées, au niveau desquelles étaient notées des erreurs de réplication et une instabilité des microsatellites hétérogènes chez un même malade; le gène hPMS2 était altéré chez deux malades, le gène $h M L H l$ chez un seul. Quelques conclusions peuvent être tirées de cette étude : tout d'abord, le syndrome de Turcot peut être associé à deux types différents de défauts génétiques de la lignée germinale touchant, soit le gène $A P C$, soit les gènes de la réparation de l'ADN; en second lieu, ce syndrome est rare, puisqu'il n'a été trouvé que che'z environ 1,5\% des familles inscrites sur les registres des cancers colorectaux familiaux étudiés. Il faut noter cependant que le facteur de risque des tumeurs cérébrales chez ces familles est très supérieur à celui de la population générale, ce qui conforte l'idée d'un syndrome et non de simple coïncidence : le risque de survenue d'un médulloblastome chez les malades ayant une mutation germinale du gène $A P C$ ' est multiplié par 92.

E.B.

1. Turcot J, Després JP, Saint-Pierre F. Malignant tumors of the central nerous system associated with familial polyposis of the colon: report of two cases. Dis Colon Rectum 1959; 2 : 465-8.

2. Olschwang S, Laurent-Puig P, Thomas (; Expression phénotypique de la polypose adénomateuse familiale. méderine/sciences 1994; 10: 4546.

3. Lynch HT, Smyrk TC, Watson P, Lanepa S], Lynch JF, Lynch PM, Cavalieri RJ, Bolland CR. Genetics, natural history, tumor spectrum, and pathology of hereditary non polyposis colorectal cancer: an updated review. Gicstroenterology 1993 . $104: 15.35-49$.

4. Thomas (G. Dix ans de recherche sur les prédispositions génétiques au développement des tumeurs. médecine/sciences 1995 ; 11 : 336.48. 